\title{
Das alte Buch in einer jungen Bibliothek - Wie geht Konstanz mit seinen Rara um? Peter Christoph Wagner
}

Die Bibliothek der Universität Konstanz ist weithin für ihre moderne Konzeption und den umfassenden Einsatz der elektronischen Datenverarbeitung sowie die Weiterentwicklung elektronischer Dienstleistungen bekannt. Aber Rara und alte Bücher - gibt es so etwas überhaupt in Konstanz? Und wenn ja, sind sie hier nicht etwa fehl am Platz? Dies mag vielleicht die erste verblüffte Reaktion auf das Lesen der Überschrift sein. Tatsächlich verfügt die Bibliothek der Universität Konstanz über einen Bestand von 19.213 Rara ${ }^{1}$, deren Erwerbung, Erschließung und Benutzung unter Wahrung der Aspekte eines bestandsschonenden Umgangs von Anfang an - das heißt seit 1964 - zu ihren Aufgaben gehörte.

Im folgenden sollen der Aufbau der Rara-Sammlung, der Umfang des Bestands sowie die Aktivitäten und der Umgang mit dem alten Buch skizziert werden.

Es war von vornherein klar, dass wir nicht in Konkurrenz zu den großen, alten Universitäts- oder Landesbibliotheken mit ihren in Jahrhunderten historisch gewachsenen reichen Schätzen an alten Büchern würden treten können. Dies war auch nicht beabsichtigt; die Konzeption war eine andere: Zum einen sollten Anschauungsobjekte für den akademischen Unterricht gewonnen werden; zum anderen sollte mit einer Sammlung des alten Buches der Verankerung der Bibliothek als Stätte des präsenten Wissens in einer historischen Tradition und Kontinuität Rechnung getragen werden. Die Bewahrung eines historischen Erbes sollte ein Gegengewicht zu den raschen Veränderungen der Gegenwart bilden und das Bewusstsein dafür schärfen, dass die Leistungen der Gegenwart auf den Erkenntnissen der Vergangenheit beruhen und aufbauen.

\section{Konzeption}

Für die Konzeption des Rara-Bestands in Konstanz gilt folgender Hauptgrundsatz: die Zeitgrenze von 1800. Alle Bücher mit Erscheinungsjahr bis 1800 kommen ohne Ausnahme zu den Rara. Nach 1800 Erschienenes kommt dann zu Rara, wenn es sich um literarische Erstausgaben, um besonders sel-

1 Stichtag: 21.10.2003 - diese Zahl setzt sich zusammen aus 14.602 Büchern mit R-Signatur und 4611 Büchern mit 5er-Signatur, dazu s. unten S. 20. 
tene bzw. wertvolle Werke, um bibliophile Ausgaben mit Buchschmuck oder kostbaren Illustrationen handelt, also um Werke, die auch für Sammler und Liebhaber von Interesse wären und somit diebstahlsgefährdet sind. Als ein Beispiel sei hier das reich illustrierte Werk „Beiträge zur Geschichte der Niederösterreichischen Statthalterei. Die Landeschefs und Räthe dieser Behörde von 1501 bis 1896; Mit den Wappen u. zahlreichen Lichtdruckbildnissen der Landeschefs" genannt, das in Wien im Jahre 1897 erschien (Signatur: R 56/51) und das in keiner anderen Bibliothek in Baden-Württemberg vorhanden ist. Auch die "Gartenlaube“ (R 56/726) sowie der „Simplicissimus“ (R 56/671) haben daher hier ihren Platz.

Der Rara-Bestand ist der einzige nicht fachlich-systematisch aufgestellte Buchbestand unserer Bibliothek und nimmt auch dadurch eine Sonderstellung ein. Unter Berücksichtigung der Formattrennung wurde von Anfang an eine Numerus-currens-Aufstellung unter der Signaturengruppe „R“ konzipiert. Der Buchstabe $R$ steht für Rara. Die Normalformate - es gelten die Höhenmaße von 14 bis $28 \mathrm{~cm}$ - werden in Jahresscheiben der Akzessionierung angeordnet. Begonnen wurde im Jahre 1968, was bedeutet, dass alle in diesem Jahr bearbeiteten Rara eine Signatur zwischen R 68/1 und R 68/192 erhielten. Entsprechend dann im Jahre 1969 die Signatur R 69/ 1ff. Inzwischen sind wir bei R 103/15 für das im Jahre 2003 zuletzt katalogisierte Werk angelangt. Kleinformate, die also kleiner sind als $14 \mathrm{~cm}$, werden unter R 58/... numerus currens aufgestellt; Großformate (28 bis $45 \mathrm{~cm}$ ) unter R 56/... und Liegeformate (über $45 \mathrm{~cm}$ ) unter R $57 / \ldots$

Im Laufe der Zeit machte sich bemerkbar, dass für zwei Probleme eine Lösung fehlte: Sonderformate wie Buchkunstobjekte, Grafiken, alte Filmrollen etc. waren für die Aufstellung zwischen den Büchern zu sperrig. Dafür wurde im Jahre 1996 eine neue Signaturengruppe R 54/... eingeführt. Am augenfälligsten dabei ist eine Konservendose mit 30 Krash-copy und Foto-Postkarten im Saitling des Künstlers Andreas J. Weigoni von 1991 (R 54/9). Jüngstes Beispiel ist eine Filmrolle (R 54/21) mit dem sechsminütigen Filmexperiment „Anémic cinéma" von Marcel Duchamp aus dem Jahre 1926. Hierbei handelte es sich um einen Wunsch der Kunsthistorikerin Friederike Wappler, die diesen Film für ihr Foucault-Seminar im SS 2003 benötigte.

Außerdem fehlte eine Lösung für solche Bücher, die zwar abgesondert vom Freihandbereich aufbewahrt werden sollten, die aber andererseits nicht so wertvoll waren, dass sie überhaupt nicht ausgeliehen werden könnten - ein Problem, welches sich an einer Magazin-Bibliothek überhaupt nicht stellt. So kam es auf Beschluss der Fachreferentensitzung im Jahre 1996 zur Schaffung einer zweiten Rara-Reihe, in der die Bücher systematische Signatur mit vorangestellter Standortkennziffer 5 tragen. Daher wird diese Rara-Sammlung in unserem Jargon als „5er Reihe“ bezeichnet. In einer mehr als zweijährigen 
Kampagne durchforstete ein Mitarbeiter, der Sprachwissenschaftler und Schriftsteller Günter Posch ${ }^{2}$, sowohl die bisherigen Rara-Bestände als auch den Freihandbereich von fast zwei Millionen Bänden auf der Suche nach Werken dieser zweiten Kategorie. Ein gewisser Schwerpunkt lag dabei auf Büchern mit Erscheinungsjahr zwischen 1800 und 1850, denn an der Zeitgrenze von 1800 für die Aufstellung unter R wurde und wird unverändert festgehalten.

\section{Geschäftsgangsorganisation}

Wie ging nun die Erwerbung alter Bücher vor sich? Wie wurde die Einbindung in den Geschäftsgang geregelt und welche Zuständigkeitsregelungen getroffen? Es wurde die Grundsatzentscheidung gefällt, keine eigene organisatorische Abteilung Alte Drucke zu bilden, sondern die einzelnen Arbeitsschritte parallel zur Bearbeitung des Neuzugangs in die Organisationsstruktur einzubinden: Die Anschaffungsauswahl obliegt somit grundsätzlich allen Fachreferenten, die entscheiden, ob sie ein altes Buch, das in ihr Fächerspektrum fällt, für den Rara-Bestand erwerben wollen. Dafür steht auch kein zentraler Finanztitel zur Verfügung, sondern die Finanzierung erfolgt aus dem jeweiligen Fachkontingent, aus dem auch die neuen Bücher zu bezahlen sind. Akzession und Katalogisierung werden in den nach Fachgruppen thematisch gegliederten Teams der Buchbearbeitung vorgenommen. Lediglich die Vergabe der Signatur erfolgt zentral in dem Team G, das auch für die Werke der Klassischen Philologie zuständig ist. Dort liegt auch die Zuständigkeit für Korrekturen der Titelaufnahme oder der Signatur.

Aufgestellt werden die Rara im abgeschlossenen Rara-Raum; somit sind sie der einzige Bestand in der Konstanzer Universitätsbibliothek, der nicht frei zugänglich ist. Von 1968 bis Mai 2003 war dies der durch ein Gitter abgeschlossene und mit einem Intervallschalter beleuchtbare klimatisierte Raum hinter dem AV-Bereich der Mediothek, was man durchaus als Kuriosum werten kann! Organisatorisch gehört der Rara-Raum zur Mediothek und wird im Rahmen der Benutzungsabteilung vom Personal des Sachgebietes Mediothek unter einer Sachgebietsleiterin verwaltet und bedient. Ein Fachreferent des höheren Dienstes ist seit 1990 zusätzlich mit der Bestandserhaltung und allen damit zusammenhängenden Fragen als Querschnittsaufgabe betraut. Seine Vorschläge werden im Benehmen mit den Mitarbeiterinnen und Mitarbeitern der Benutzungsabteilung umgesetzt. Diese Zusammenarbeit erfolgt unhierar-

2 Dieser hat auch den Beitrag über die Bibliothek der Universität Konstanz für das „Handbuch der historischen Buchbestände in Deutschland“ Bd. 8 (1994), S. 98-101 verfasst. 
chisch und entsprechend dem Konstanzer Betriebsklima konfliktfrei und harmonisch.

Der Bezug des Bibliothekserweiterungsbaus im Mai 2003 brachte auch für die Rara eine einschneidende Änderung: Der größte Teil, nämlich die Signaturengruppe R, zog in die neue Klimakammer um, die im Inneren des Anbaus als rundum geschlossener Betonraum liegt und mit Kompaktregalen ausgestattet ist. Die Klimaanlage gewährleistet $18 \mathrm{Grad}$; die Luftfeuchtigkeit ist auf $50 \%$ eingestellt. Die Intervallschalter verbreiten nur ein dämmriges Licht ${ }^{3}$. Die zweite Rara-Reihe, die „5er-Reihe“, verblieb dagegen im alten Rara-Raum. Nur so konnte nämlich Platz für Neuzugänge gesichert werden.

Für die Benutzung der Rara-Werke dient nach wie vor der Lesesaal der Mediothek. Auf Wunsch werden dort die Rara aus beiden Räumen unter Aufsicht zur Präsentnutzung bereit gestellt.

Die bibliographische Erschließung erfolgt wie bei allen unseren Büchern durch die Katalogisierung im Verbundkatalog des Südwestdeutschen Bibliotheksverbundes ${ }^{4}$, aus dem dann die Datenabzüge ins lokale System ${ }^{5}$ erfolgen; d.h. alle Rara sind über inre Autoren, Titel oder Signaturen recherchierbar. Eine gezielte Suche ist dagegen nur im OPAC möglich, wo man die Autor- oder Titelsuche mit der Aufstellung bei Rara verknüpfen kann. So kann man z.B. nach den Werken Senecas im Rara-Bestand suchen mittels Eingabe von „Seneca" im Personenfeld und der Verknüpfung mit dem Fachgebiet „rar" und erhält 15 Treffer.

\section{Bestandsaufbau der Rara-Sammlung}

Da keine geschlossenen Altbestände oder größeren Sammlungen als Geschenk, Vermächtnis oder Kauf den Grundstock zur Rara-Sammlung in Konstanz bildeten, mussten die Werke mehr oder weniger einzeln im Laufe der Jahre erworben werden - ein durchaus mühseliges Geschäft. Grundlage für die Fachreferenten bildeten die Kataloge deutscher und ausländischer Antiquariate, die aufmerksam durchforscht werden mussten.

Im Falle der Versteigerung der Fürstlich-Fürstenbergischen Hofbibliothek konnte Konstanz sogar bei den Auktionen mitbieten und in den Jahren 1991

3 Während der mehrjährigen Planungsphase war das Bauamt von Seiten der Bibliothek auf die Einhaltung von ISO 11799 für die Anlage der neuen Klimakammer hingewiesen worden.

4 http://www.bsz-bw.de/CGl/cgi-bin/opacform.cgi

5 http://www.ub.uni-konstanz.de/koala/ 
bis 2001 insgesamt 38 Werke mit Bezug zu Konstanz und der Region ersteigern!

Ergänzt wurden die Käufe in einigen Fällen durch Geschenke und Sponsoring. Die prächtigsten Beispiele für Geschenke sind die „Erdbeer-Initiale“ von 1500 (H 57/3), welche uns im Jahre 1997 Botschafter a. D. Dr. Wolfram Dufner schenkte $^{7}$, sowie das Faksimile des Miniaturstundenbuchs von Lorenzo de Medici (R 55/283), welches im Jahre 1995 vom damaligen Wissenschaftsminister Klaus von Trotha der Bibliothek geschenkt wurde ${ }^{8}$.

Gesponsert wurden uns drei wichtige Werke:

- die „Konstanzer Handschrift“ von $1416 / 17^{9}$, deren Erwerb im New Yorker Antiquariatsmarkt 1985 durch die finanzielle Unterstützung der Badischen Landesbausparkasse, der Universitätsgesellschaft Konstanz, der Maggi $\mathrm{GmbH}$, der Sparkasse Konstanz sowie des Südkuriers glückte ${ }^{10}$;

- eine Hus-Flugschrift von 1525 mit dem Titel „Geistlicher Bluthandel Johannis Hussz“ (R 96/192), deren Erwerbung uns der Konstanzer Arzt Dr. Peter Ehlers im Jahre 1996 ermöglichte ${ }^{11}$;

- Schließlich bot das Jubiläum des Erwerbs des zweimillionsten Buches im Bestand der Konstanzer Universitätsbibliothek die Gelegenheit, die Universitätsgesellschaft um das Sponsoring des 2.000.001. Buches zu ersuchen, wozu sich diese gerne bereit erklärte. Es musste nicht lange nach einem repräsentativen Werk gesucht werden. Im Kontakt mit den Kunsthistorikern wurde der Wunsch nach dem Werk "La méthode graphique dans les sciences expérimentales“ von Étienne-Jules Marey aus dem Jahre 1878 genannt, das gerade auf dem Antiquariatsmarkt angeboten wurde und sehr gut in das augenblickliche Forschungs- und Lehrprogramm des Kunsthistorikers Steffen Bogen passen würde! Die Erwerbung glückte, und Mareys Buch wurde in einer öffentlichen Feier am 18. Juni 2002 von der Universitätsgesellschaft der Bibliothek übergeben und von Steffen Bogen anhand von vier Plakaten erläutert, die Mareys Porträt zeigten sowie seine Grafiken und Messmethoden

6 Anm.: vgl. unten S. 29.

7 vgl. unten S. 32.

8 Wagner, Peter Christoph, Rara-Ausstellung, in: Bibliothek aktuell 66 (1995) S. 19f. = http://www.ub.uni-konstanz.de/kops/volltexte/2002/763/; ders. in: uni-info 237 (1995), S. 30.

9 vgl. unten S. 31.

10 http://www.ub.uni-konstanz.de/_spons/obj1.htm

11 http://www.ub.uni-konstanz.de/_spons/obj2.htm; vgl. auch Herbert-E. Probst, Private Unterstützung. Die Universitätsbibliothek bekam wertvolle Druckschrift über Jan Hus geschenkt, in: uni-info 248 (1996) S. 40. 
zur Visualisierung raum-zeitlicher Zusammenhänge ${ }^{12}$. Dies war außerdem auch eine der spektakulärsten Aktivitäten im Umgang mit unseren Rara.

Im Laufe der Zeit gelang es, einen Bestand aufzubauen, der eine gewisse Ausgewogenheit in dem Sinne aufweist, dass alle Epochen der Druckgeschichte und alle Literaturgattungen vertreten sind; außerdem auch die wichtigsten alten Druckorte sowie alle größeren Länder Europas. Ein Schwerpunkt wurde dabei auch auf den Erwerb eines regionalbezogenen Bestands gelegt.

\section{Umfang des Bestands}

Zur Skizzierung des Bestandsumfangs sollen im folgenden exemplarische Werke für die verschiedenen Gattungen aufgeführt werden:

Die wichtigsten antiken Autoren des Klassischen Altertums sind alle vertreten, in der Regel sogar in mehrfachen Editionen und Kommentaren; so gibt es zu Aristoteles 14 Ausgaben, zu Cicero sogar 44 Ausgaben. Um nur willkürlich eine einzelne Klassiker-Ausgabe hier herauszugreifen: eine Livius-Ausgabe des Jahres 1628 aus Frankfurt am Main, die von dem niederländischen Humanisten Franciscus Modius herausgegeben und von dem Poeten und Juristen Janus Gruterus kommentiert worden ist (R 56/506). An diese Edition sind übrigens drei weitere Kommentare zu lateinischen Autoren angebunden, die 1627 in Frankfurt am Main beim selben Drucker J. Fischer erschienen sind. Nach Ausweis des Verbundkatalogs sind diese Kommentare nur in Konstanz vorhanden, während die Livius-Ausgabe nur noch in Dresden vorhanden ist!

Auch von den für den französischen Thronfolger „,in usum Delphini“ bearbeiteten Klassikern haben wir eine Ausgabe, und zwar die „Epitoma“ des Justinus, erschienen 1677 (R 103/5); laut Verbundkatalog nur in Konstanz vorhanden ${ }^{13}$.

Die Humanisten sind im Konstanzer Rara-Bestand außer mit den KlassikerEditionen auch mit eigenen Werken vertreten; stellvertretend seien genannt der Humanist und Lexikograph Ambrogio Calepino (1435-1511) mit seinem fünfsprachigen Wörterbuch „Pentaglottos“, das 1546 in Antwerpen erschien (R 73/59), und der Humanist Jakob Henrichmann (1482-1561) mit seinen "Grammaticae institutiones“, Augsburg 1539 (R 73/163). Beide Werke sind laut Verbundkatalog nur in Konstanz vorhanden.

12 vgl. die Abbildungen auf unserer Homepage: http://www.ub.uni-konstanz.de/ zweimillionen.htm

13 Anm.: Wagner, Peter Chr., In usum Delphini, in: Bibliothek aktuell 69 (1997) S. $31=$ http://www.ub.uni-konstanz.de/kops/volltexte/2002/766/ 
An gelehrten Werken über das Altertum sei hier nur das numismatische Werk „Notitia elementaris numismatum antiquorum illorum ..." von Erasmus Fröhlich aus dem Jahre 1758 (R 93/6) vorgestellt oder die Beschreibung der Geographie und Topographie Italiens im Altertum „Italia antiqua“ von Philipp Clüver, die wir in der Wolfenbütteler Ausgabe von 1659 besitzen (R 89/194).

Breiten Raum nehmen auch Bibelausgaben und theologische Literatur ein: hervorgehoben sei hier eine prachtvolle sechsbändige Vulgata-Ausgabe mit goldgeprägtem Rückenschmuck aus Paris (1693-1706) mit einem schönen Kupferstich des HI. Hieronymus (R 56/38). Enthalten sind auch die Briefe und Kommentare sowie eine Lebensbeschreibung des HI. Hieronymus. In BadenWürttemberg ist diese Ausgabe außer in Konstanz nur in Heidelberg vertreten. Vom HI. Bruno, dem Gründer des Kartäuserordens, haben wir eine Gesamtausgabe seiner Werke („Opera omnia“), die 1611 in Köln gedruckt wurde (R 56/106). Ein erst jüngst bei den Versteigerungen der Fürstlich-Fürstenbergischen Hofbibliothek erstandenes Werk ist das „Opus super sentencias valde egregium in disciplina theologie cunctis proficere volentibus..." von Nicolas Denisse, Rouen 1506 (R 99/74).

Leichenpredigten besitzt Konstanz dagegen nur drei. ${ }^{14}$

Philosophische Literatur ist ebenfalls zahlreich im Konstanzer Rara-Bestand vertreten; herausgegriffen seien hier nur die „Specimina philosophiae“, die lateinische Übersetzung von Descartes „Discours de la méthode“, die 1672 in Amsterdam bei Elzevir in zweiter Auflage erschien (R 68/88). Der Verbundkatalog verzeichnet Konstanz als einzige besitzende Bibliothek.

Von Geschichtswerken bzw. historischer Literatur, die in unserem RaraBestand gesammelt sind, seien hier nur zitiert: die „Historia del emperador Carlos Quinto“, Madrid 1675 (R 56/27) von Prudencio de Sandoval (1560?1620); die zehnbändige „Chronologische Geschichte Böhmens unter den Slaven“ von Frantisek Pubicka, die in Leipzig in den Jahren 1770 bis 1801 erschien (R 96/344), oder die Akten des Westfälischen Friedens, die „Acta Pacis Westphalicae“ (R 56/49), die erst über 80 Jahre später (1734-1740) in Hannover in sieben Bänden gedruckt wurden. Als ein Werk zur orientalischen Geschichte und historischen Topographie des Vorderen Orients, von Kleinasien über Mesopotamien bis Arabien, lässt sich die „Umbstaendliche und eigentliche Beschreibung von Asia“ von Olfert Dapper zitieren, 1681 in Nürnberg auf Deutsch erschienen und mit schönen Stadtansichten illustriert (R 56/1181).

14 R 56/446[angebunden]; R 84/240; R 74/144[angebunden]. 
Historisch-geographische Karten und Atlanten sind demgegenüber nur spärlich vertreten: Lediglich ein Atlas des 17. Jahrhunderts (R 56/1104) ${ }^{15}$ und vier Atlanten aus dem 19. Jahrhundert sind vorhanden, die durch zwei Faksimiles ergänzt werden. Alte Karten sind nur als Beilagen in historischen Werken vorhanden. Dieser Mangel ist angesichts der Tatsache, dass es aus Konstanz eine Weltkarte des 15. Jahrhunderts gibt, umso empfindlicher: 1448 hat der Benediktinermönch Andreas Walsperger diese Weltkarte in Konstanz im Format 58,7 x $75 \mathrm{~cm}$ gezeichnet ${ }^{16}$. Immerhin haben wir sie als Faksimile (K 85/2).

Was in der Konstanzer Rara-Sammlung gänzlich fehlt, ist ein alter Globus.

Von den juristischen Werken im Konstanzer Rara-Bestand seien die folgenden drei hier vorgestellt: der „Tractatus et synopsis totius iuris feudalis“, ein Kompendium des Lehnsrechts, von Heinrich von Rosenthall (R 56/638), ein zweibändiges Werk mit reichem Einbandschmuck im Stil der Spätrenaissance, das 1597 und 1600 in Frankfurt am Main erschien; ferner die Reichskammergerichtsordnung des Reichshofgerichts in Rottweil, 1535 in Frankfurt am Main von Chr. Egenolff gedruckt (R 56/307); sowie der „Code Napoleon als Badisches Landrecht“", Karlsruhe 1809 (R 99/84).

Aus dem Bereich der naturwissenschaftlichen Literatur sei hier Kaspar Bauhin's Pflanzensystematik „Pinax theatri botanici sive Index in Theophrasti, Dioscoridis, Plinii et Botanicorum ... opera" angeführt (R 88/223). Wir besitzen es nicht in der Erstausgabe von 1623, sondern in einer späteren Auflage aus dem Jahre 1671. Immerhin sind wir mit dieser Ausgabe die einzige besitzende Bibliothek in Baden-Württemberg. Bauhin führte die binäre Nomenklatur ein; sein Werk blieb bis zu Linnés Systematik grundlegend. Es ist mit zahlreichen Holzschnitten ausgestattet, welche die Pflanzen detailgenau wiedergeben. Mit diesem Werk ist übrigens auch Basel als bedeutender alter Druckort in unserer Bibliothek vertreten.

Zwei Beispiele aus der in Konstanz vorhandenen medizinischen und pharmazeutischen Literatur: zum einen „Die medizinische Eröffnung“ (R 89/196), ein zweibändiges, 1724 und 1726 in Leipzig erschienenes Werk zur Diagnose und Therapie der Krankheiten, von Georg Ernst Stahl (1660-1734), Professor für Medizin und Leibarzt des preußischen Königs Friedrich Wilhelm I.; Stahl gilt

15 Es handelt sich um eine Zusammenstellung der Karten des französischen Kartographen Jean-Baptiste Bourguignon d'Anville aus dem Geschichtswerk „Histoire romaine" von Charles Rollin (1738-1748). Da ein Titelblatt fehlt, ist das Erscheinungsjahr ungewiss. Der Rückentitel „Atlas de géographie ancienne“ gehört eher zu dem späteren Einband.

16 Cod. Vat. Pal. Lat. 1362b; vgl. zuletzt Huth, Volkhard, Zeit ist mit dem Himmel entstanden. Auf den Spuren archaischer Zeitauffassung (=Konstanzer Universitätsreden 213) (2003) S. 6f. Abb 1 mit Lit. 
als Begründer des Animismus, wonach die Krankheiten durch den Zustand der Seele verursacht würden. Übrigens ist „Die medizinische Eröffnung“ laut Verbundkatalog nur in Konstanz vorhanden. Zum anderen der „Tractado de las drogas, y medicinas de las Indias Orientales“ (R 79/168), eine Studie über die Heilpflanzen in Indien von Cristobal de Acosta (ca. 1515 bis ca. 1592), erschienen in Burgos im Jahre 1578 und ebenfalls nur in Konstanz vorhanden.

Reiseberichte sowie Forschungsreiseberichte sind ebenfalls vorhanden, z.B. die "Reise in Deutschland, der Schweiz, Italien und Sicilien“ von Friedrich Leopold Graf zu Stolberg, erschienen 1794 in Königsberg (R 88/233) oder Carsten Niebuhrs „Beschreibung von Arabien“ von 1772 (R 80/249).

Im Anschluss daran sei auch hingewiesen auf die alpinistischen Bergsteigererlebnisse von Edward Whymper "Scrambles amongst the alps", in der seltenen Erstausgabe London 1871 (R 88/376), mit der Schilderung der dramatisch verlaufenden Erstbesteigung des Matterhorns.

Aus dem Bereich slawischer Literatur seien hier zwei Werke vorgestellt: Ein kirchenslawisches Werk besitzt Konstanz mit der „Lestvica“ (R 56/453), der Übersetzung der im Original griechischen „Himmelsleiter" des Johannes Klimakos, erschienen in Moskau im Jahre 1648 und It. Verbundkatalog nur in Konstanz vorhanden. Es ist eine mönchische Anleitung, die das orthodoxe Mönchstum stark beeinflusst hat. Johannes Klimakos war im 7. Jahrhundert Abt des Sinaiklosters. Sehr seltene Erstausgaben aus neuerer Zeit besitzen wir mit vier Werken (R 101/13 - R 101/16) von Mark Aleksandrovic Aldanov (1886-1957), der als Begründer des russischen historischen Romans gilt. Diese unter dem Obertitel „Myslitel" (=der Denker) zusammengefaßten vier Werke bilden einen Zyklus historischer Romane zur Französischen Revolution, der Napoleonischen Zeit und den Befreiungskriegen. Sie erschienen 1923-1927 in Berlin und Paris ${ }^{17}$.

Orientalistische Studien sind ebenfalls in unserem Rara-Bestand vertreten: für die hebräische Philologie lässt sich z.B. der „Thesaurus grammaticus linguae sanctae Hebraeae" (R 80/225) des Schweizer Hebraisten Johann Buxtorf anführen (Basel 1629); für die Arabistik z.B. das „Lexicon Arabicvm“ (R 94/1) von Franciscus Raphelengius ( Leiden 1613), dem Schöpfer der arabischen Drucktypen in Holland ${ }^{18}$. Auch alte Editionen arabischer Klassiker können in diesem Zusammenhang genannt werden, z.B. die „Al-Agurrumiya“ (,Grammatica ara-

17 Wagner, Peter Chr., Seltene russische Erstausgaben vor dem Zerfall gerettet, in: Bibliothek aktuell 76 (2002) S. 20 = http://www.ub.uni-konstanz.de/kops/ volltexte/2002/775/

18 zu Raphelengius vgl. zuletzt Hamilton, Alastair: Arab culture and Ottoman magnificence in Antwerp's golden age (2001) S. 71-76. 
bica“) von Ibn-Agurrum (1273-1323), die 1617 in Leiden erschien (R 80/220). Besonders bemerkenswert ist in dieser Hinsicht die "Chronologica et astronomica elementa" von Al-Fargani (ca. 800 bis ca. 861) in der lateinischen Übersetzung von Jakob Christmann, dem ersten Arabistik-Professor in Deutschland, die 1590 in Frankfurt am Main erschien (R 81/91). Die Verbreitung des ptolemäischen Weltbildes im Mittelalter ist ganz entscheidendend der arabischen Fassung von Al-Fargani zu verdanken, die im Mittelalter ins Lateinische zurückübersetzt wurde ${ }^{19}$.

Im Bereich der Belletristik wurde mit besonderem Nachdruck die Erwerbung von Erstausgaben betrieben: Von Gottfried Benn haben wir immerhin 13 Erstausgaben: „Gehirne“ (1916); „Gesammelte Prosa“ (1928); „Das Unaufhörliche“ (1931); „Nach dem Nihilismus“ (1932); „Der neue Staat und die Intellektuellen“ (1933); „Kunst und Macht“ (1934); „Statische Gedichte“ (1948); „Drei alte Männer“ (1949); „Fragmente“ (1951); „Die Stimme hinter dem Vorhang“ (1952); „Destillationen“ (1953); „Aprèslude“ (1955); „Reden“ (1955).

Auch von Franz Kafka haben wir mehrere Erstausgaben: „Der Heizer“ (1913 R 82/208), „Die Verwandlung“ (1915 - Sign. R 101/17), „Das Urteil“ (1916 Sign. R 82/209), „Der Prozess“ (1925 - Sign. R 69/168), „Das Schloss“ (1926 Sign. R 101/18), „Beim Bau der Chinesischen Mauer“ (1931 - R 89/88), „Vor dem Gesetz" (1934 - R 82/165) sowie die von Max Brod 1935-1937 in sechs Bänden herausgegebenen „Gesammelte(n) Schriften“ (R 101/19).

Von James Joyce haben wir den „Ulysses“, als 947. von 1000 Exemplaren der Erstausgabe von 1922 (R 100/14). Diese antiquarische Erwerbung glückte dem zuständigen Fachreferenten Dr. Uwe Jochum erst vor wenigen Jahren. Außer in Konstanz ist in Deutschland nur noch in Göttingen ein Exemplar der Erstausgabe vorhanden! Eine weitere kostbare Erwerbung aus jüngerer Zeit ist „The life and opinions of Tristram Shandy” von Laurence Sterne in der 2. Ausgabe in den neun Bänden von 1760-1767 (R 99/78). Diese Ausgabe ist nur in Konstanz vorhanden.

Um auch ein Werk der französischen Literatur zu zitieren: die „Fables nouvelles,, von Antoine Houdar de La Motte in der Erstausgabe von 1719 (R 68/94). An italienischer Belletristik kann ein Werk von Boccaccio angeführt werden allerdings nicht das berühmte Decamerone, sondern die „Genealogia deorum gentilium“, die 1472 zuerst auf Latein erschien und erst später ins Italienische übersetzt wurde. Wir haben die lateinische Ausgabe von 1511 aus Paris (R 56/245), als einzige Bibliothek in Baden-Württemberg.

19 vgl. dazu http://www.ub.uni-konstanz.de/fi/rara/index.htm\#1200_Jahre_Al-Fargani 
Im Anschluss an die Belletristik seien die Malerbücher aufgeführt, die ebenfalls vertreten sind; genannt seien hier nur die "Contes de Boccace“ mit den Zeichnungen Chagalls aus dem Jahre 1950 (R 56/517-24) oder eine rezente Erwerbung, die „Aquarelle zu Mozarts Zauberflöte“ von Max Slevogt, bei Piper in München 1920 erschienen (R 56/1334).

Sekretierte Literatur ${ }^{20}$ die man früher gerne weggeschlossen hat, weist der Konstanzer Rara-Bestand nur in geringerem Maße auf. Hauptsächlich handelt es sich um NS-Literatur wie z.B. den "Völkischen Beobachter" (R 55/166) oder Hitlers Rede im Bürgerbräukeller von 1925 (R 92/81). Eigentliche „Sittengeschichten" haben wir nur sieben, z.B. die "Sittengeschichte des Hafens und der Reise. eine Beleuchtung des erotischen Lebens in der Hafenstadt, im Hotel, im Reisevehikel“, 1927 (R 85/138), die mit ihren circa 200 ein- und mehrfarbigen Illustrationen und Kunstbeilagen (sic!) wohl weniger wegen moralischer Gefährdung der Allgemeinheit als wegen Klaugefährdung zum eigenen Schutz sekretiert sein muss!

Rara mit Regionalbezug bilden einen Schwerpunkt: Daher hatten wir bei der Versteigerung der Fürstlich-Fürstenbergischen Hofbibliothek ein besonderes Interesse an den Werken mit Bezug zu Konstanz und der Region. Von den insgesamt 38 ersteigerten seien hier hervorgehoben: das oben erwähnte ${ }^{21}$ theologische Werk von Denisse (R 99/74), das ursprünglich im Besitz des Minoriten-Konvents von Konstanz war; das "Directorium divini officii“" von 1820 aus dem Kloster Salem (R 58/557); das anlässlich des Todes von Joseph II. im Jahre 1790 in Konstanz gedruckte Trauergedicht "Die Eitelkeit“ (R 100/22); oder die Gedichtsammlung des Schweizer Dichters Johann Jakob Rüttlinger in Toggenburger Mundart, 1835 in Lichtensteig gedruckt und ehemals im Besitz des Freiherrn von Lassberg (R 99/70).

Ein thematischer Schwerpunkt bei den Regionalbezügen wurde mit der Erwerbung der Werke von und über Jan Hus und die Hussiten gebildet. Es entstand eine nahezu vollständige Sammlung der Originalquellen und der Sekundärliteratur vom frühen 16. Jahrhundert bis zur Gegenwart, unter Einschluss der Rezeption in den verschiedenen geistesgeschichtlich-politischen Strömungen der tschechischen Literatur (Nationalisten, Kommunisten, Devotionalliteratur). Insgesamt handelt es sich um über 600 Titel, die allerdings nur zu einem kleinen Teil - nämlich 12 - bei Rara, in ihrer überwältigenden Mehrheit dagegen an den in Frage kommenden Systemstellen gsf 938 - 942 im Freihandbereich stehen.

20 vgl. diesbezüglich die Ausstellung „Der Giftschrank“. Erotik, Sexualwissenschaft, Politik und Literatur - „Remota“: die weggesperrten Bücher der Bayerischen Staatsbibliothek (München 2002).

21 Vgl. oben S. 25. 
Alte Reiseführer der Bodensee-Region bilden einen weiteren Schwerpunkt, der etwa 60 Werke umfasst. Der älteste Bodenseeführer in unserem Bestand ist von Gustav Schwab aus dem Jahre 1827 (R 68/112).

Wegen des Regionalaspekts werden auch die Werke der Annette von DrosteHülshoff gesammelt, von denen wir 15 Ausgaben im Rara-Bestand besitzen. Dazu hat der damalige Fachreferent für Germanistik eine Broschüre verfasst ${ }^{22}$.

Ebenfalls zur Regionalsammlung sind die Schriften des wortgewaltigen Barockpredigers Abraham a Sancta Clara zu zählen, da er aus Kreenheinstetten im Kreis Sigmaringen stammt, wo er im Jahre 1644 als Hans Ulrich Megerle geboren wurde. Seine Werke haben wir, in Gesamt- sowie in Einzeleditionen, in 24 Ausgaben.

Nicht ganz so berühmt ist ein weiterer geistlicher Barock-Schriftsteller: Laurentius von Schnüffis (1633-1702) aus Schnifis in Vorarlberg, der als Kapuzinermönch in Konstanz gesungen und gedichtet hat. Von ihm haben wir fünf Werke im Rara-Bestand: „Mirantisches Flötlein, oder Geistliche Schäfferey“ (R 86/6), „Mirantische Wald-Schallmey“ (R 86/50), „Mirantische Maul-Trumel“ (R 87/11), „Futer über die Mirantische Maul-Trummel“ (R 87/11[angebunden]) und „Vil-färbige Himels-Tulipan“ (R 90/97). Zuletzt wurde Laurentius von Schnüffis in einer Ausstellung der Österreichischen Nationalbibliothek gewürdigt ${ }^{23}$.

Hinsichtlich des Regionalbezugs ist auch erwähnenswert, dass im RaraBestand 80 in Konstanz erschienene Werke vorhanden sind, die eine Zeitspanne von 1596 („Epitome sacramentorum“ von Lorenzo Pezzi) bis 2001 („Floral artifacts. Postkarten“ der Künstlerin Ruth Handschin) umfassen.

Wenn auch alle Epochen der Druckgeschichte im Konstanzer Rara-Bestand vertreten sind, so liegt doch ein gewisser Schwerpunkt auf dem 17. und 18. Jahrhundert. Die Inkunabelzeit ist deutlich unterrepräsentiert: Die „Pharsalia“ von Lucanus, im Jahre 1477 in Venedig von Guerinus gedruckt, ist unsere einzige vollständige Inkunabel (R 56/435). Hinzu kommen noch ein Blatt aus den "Constitutiones“ des Papstes Clemens V., in Venedig 1491 gedruckt $(\mathrm{H}$ 55/1) sowie zwei Blätter aus Schedels Weltchronik von 1493 (Blatt CCXL und CCXLI), passenderweise mit der handkolorierten Ansicht von Konstanz ( $R$ 55/272) - als Glasbild gerahmt! Dem Vermerk auf der Rückseite zufolge handelt es sich um das Geschenk eines Antiquariats aus dem Jahre 1965. Viele Jahre lang hing dieses "Bild“ als Zimmerschmuck in der Direktion, bis es

22 Bergmann, Robert, Annette von Droste-Hülshoff (Konstanz 1988).

23 Brodl, Michaela, Der verbotene Blick. Erotisches aus zwei Jahrtausenden (2002) S. $123 \mathrm{Nr} .103-105$. 
schließlich 1995 der ständigen Lichtexposition entzogen und im schützenden Dunkel des Rararaums deponiert wurde!

An Frühdrucken aus der Zeit von 1500 bis 1550 haben wir immerhin 88 Bände. Darunter sind fast alle alten Druckorte vertreten: Augsburg (R 77/99), Basel (R 56/85), Strassburg (R 56/269), Frankfurt am Main (R 56/307), Köln (R 70/146), Leipzig (R 73/75), Ingolstadt (R 70/34, Wien (R 70/3), Venedig (R $56 / 352$ ), Bologna (R 56/160), Mailand (R 71/80), Rom (R 56/351), Lyon (R 56/348), Paris (R 56/1205), Utrecht (R 72/72), Antwerpen (R 73/59).

In diesem Zusammenhang ist hervorzuheben, dass im Konstanzer RaraBestand 18 Aldinen, also Drucke von Aldus Manutius und seinen Nachfolgern aus Venedig, vertreten sind.

Außer den Druckwerken besitzt Konstanz sogar einige Handschriften und Handschriftenfragmente, was mit gewissem Stolz zu vermelden ist. Es handelt sich um acht Stück, die nachfolgend alle genannt seien:

- Das älteste Stück ist ein bisher noch nicht erfasstes rubriziertes Pergamentblatt, bei dem es sich nach freundlicher Auskunft von Dr. Brigitte Hotz, Konstanz, um ein Fragment aus einer kommentierten Sammlung päpstlicher Dekretalen handelt, konkret um Compilatio IV Liber II Tit. XI Cap. 1 (Textende) bis Liber III Tit. I Cap. 1 (Textanfang) ${ }^{24}$. Diese Compilatio ist nach Friedberg nach dem 4. Laterankonzil von 1215 anzusetzen.

- Aus der Konzilszeit haben wir die „Konstanzer Handschrift“ (H 56/1), eine Sammelhandschrift, die 1416 und 1417 in Konstanz angefertigt wurde (216 $\mathrm{BI}$. $)^{25}$; sie enthält das „De Regimine principum“ von Aegidius Romanus, die "Nikomachische Ethik" von Aristoteles in der lateinischen Übersetzung von Robert Grosseteste sowie die aristotelische „Rhetorik“ in der lateinischen Übersetzung von Wilhelm von Moerbeke. Die von verschiedenen Händen in gotischer Kursive auf Papier geschriebene Handschrift hat einen lederbezogenen Holzdeckel mit Blindprägung in Form eines Andreaskreuzes; sie ist mit Schließen verschlossen. Zur Befestigung an einem Pult diente eine schwere Eisenkette, von der noch vier Glieder erhalten sind;

- besonders bemerkenswert ist eine einzelne illuminierte HandschriftenInitiale, die aus einer Handschrift herausgeschnitten war (H 57/3) und ein „M“ mit einer leuchtend roten Erdbeere zeigt. Die genaue Einordnung

24 Edition: Friedberg, Emil, Quinque Compilationes Antiquae (1882, Reprint 1956) S. $141 \mathrm{f}$.

25 L. Bösing, Eine mittelalterliche Handschrift kehrt nach Konstanz zurück, in: Konstanzer Blätter für Hochschulfragen 90, 1986, S. 5-16; http://www.ub.unikonstanz.de/fi/rara/index.htm mit Abb. 
gelang mithilfe von Fachleuten, die durch die Veröffentlichung im Internet sowie die Aufrufe in Maillisten darauf aufmerksam wurden: Die Initiale stammt nach Dr. Ulrike Bauer-Eberhardt aus einem Chorbuch aus Bergamo um 1500, während die Text- und Notenfragmente nach Dr. Felix Heinzer in den Kontext der Antiphon "In die tribulationis meae..." (3. Nokturn von Gründonnerstag) gehören ${ }^{26}$;

- das „Landrecht des Erzherzogtums Österreich“ von 1574 (nicht paginiert, 177 Bl. - Signatur: H 56/2); ein vierteiliger Justiniankommentar des Juristen Joachim Denich („Comment. in imp. Justiniani institut.“), verfasst in Ingolstadt in den Jahren 1591 und 1592 (getr. Paginierung, zusammen 435 Bl. - Sign.: H 57/1);

- eine Konstanzer Bischofschronik von Augustinus Rogg mit dem Titel „Catalogus omnium et singulorum episcoporum Vindonissensium et Constantiensium", geschrieben in Konstanz im Jahre 1641 und mit Zeichnungen der Bischofswappen (489 gezählte Seiten - Sign.: H 57/2);

- eine in deutscher Schreibschrift geschriebene Lokalchronik der Stadt Höchstädt an der Donau mit dem Titel "Geschichte und Beschreibung der Chur-Pfalz-bayr. Landgerichtshöchstaedt" von Franz Gottfried Strobel aus dem Jahre 1790. Diese wurde erst Anfang 2003 im Rara-Raum bei der Auswahl beschädigter Rara für eine Restaurierungslieferung als Handschrift entdeckt! Sie stand bei den Großformaten unter R 56/578 und umfasst 261 nicht paginierte Blätter. In Höchstädt existieren nach Auskunft des städtischen Heimatpflegers und Leiters des Heimatmuseums, Georg Strobel, noch zwei weitere handschriftliche Chroniken von F.G. Strobel deren Seitenzahlen differieren, so dass es sich vermutlich um unterschiedliche Fassungen handeln dürfte ${ }^{27}$. Strobel war Landgerichtsschreiber und Mitglied der Bayerischen Akademie der Wissenschaften ${ }^{28}$. Bekannt ist Höchstädt vor allem durch die Schlacht von Höchstädt (,the Battle of Blenheim“) im Spanischen Erbfolgekrieg am 13. August $1704^{29}$ !

26 http://www.ub.uni-konstanz.de/fi/rara/index.htm mit Abb. 1

27 Sie bilden die Grundlage für das Geschichtsbild von Höchstädt, vgl. Layer, Adolf, Höchstädt an der Donau. eine kleine Stadt mit großem Namen (1981) passim.

28 vgl. Layer, Adolf, Der Höchstädter Historiker Franz Gottfried Strobel und die Bayerische Akademie der Wissenschaften, in: JbHVDillingen 66, 1964, S. 107-112

29 Im Schloss Höchstädt findet im Jahre 2004 eine große Ausstellung der Bayerischen Verwaltung der staatlichen Schlösser, Gärten und Seen aus Anlass des 300. Jahrestags statt. 
- Schließlich gibt es noch drei handschriftliche Briefe des Antwerpener Bischofs Laevinus T. Torrentius (1525-1595), die seinem 1608 in Antwerpen gedruckten Horazkommentar (R 70/79) beigefügt waren, wie der Besitzer Gerard Nicolaas Heerkens, ein Niederländischer Arzt und Dichter (1726-1801), in einem längeren handschriftlichen Eintrag auf der Schlussseite vermerkt. Auf dem Titelblatt hat Heerkens seinen Besitzvermerk mit dem Jahr 1762 eingetragen. Diese Briefe sind bisher noch nicht erfasst.

Zur Kompensation des fehlenden Handschriftenbestands wurde von Anfang an großer Wert auf die Erwerbung von faksimilierten Codices gelegt, um einen repräsentativen Überblick über die Buchmalerei bieten zu können. Etwa 150 solcher Prachtwerke wurden im Laufe der Zeit erworben. Die Spannweite reicht vom „Vergilius Vaticanus“ (R 81/29) und der „Wiener Genesis“ (R 56/445) über das „Book of Kells“ (R 55/240), den „Codex Aureus Epternacensis“ (R 55/156) oder das Falkenbuch Kaiser Friedrichs II. „De arte venandi cum avibus" (R 69/139) bis zur "Goldene(n) Bulle“ (R 55/86), den "Petites Heures du Duc de Berry“ (R 89/167) oder dem "Gebetbuch Karls V." (R 86/56). Doch nicht nur mittelalterliche Faksimiles wurden gekauft, sondern auch neuzeitliche, insbesondere die Werke von Künstlern. An erster Stelle ist hier Leonardo da Vinci zu nennen, von dem wir 18 Ausgaben im RaraBestand besitzen. Aber auch das sogenannte Leningrader Studienbuch „Schmetterlinge, Käfer und andere Insekten“ von Maria Sibylla Merian (R 56/952) kann in diesem Zusammenhang angeführt werden. Zur Abrundung der regionalen Schwerpunkte wurden insbesondere auch die Faksimiles der Reichenauer Buchmalerei erworben. Eine unserer jüngsten FaksimileErwerbungen bildet einen besonderen Glanzpunkt: es handelt sich um die Barberini-Exultetrolle, als Belser Faksimile Edition nach dem gegen Ende des 11. Jahrhunderts in der Abtei von Montecassino entstandenen Original aus der Biblioteca Apostolica Vaticana (R 55/313). Zu den Faksimiles gibt es einen von der damaligen Referendarin Brigitte Schürmann erstellten Katalog aus dem Jahre 1988, der die damals 145 Faksimile systematisch geordnet auflistet. Leider existiert er nur als ungedrucktes Manuskript. 


\section{Umgang mit dem alten Buch}

\section{Bestandserhaltung und Bestandspflege}

Restaurierungen

Im Rahmen von Bestandserhaltung und Bestandspflege nehmen die Restaurierungen von Rara-Werken durch das Institut für Erhaltung von Archiv- und Bibliotheksgut (IfE) in Ludwigsburg den wichtigsten Platz ein ${ }^{30}$. Dieses mit den modernsten Geräten eingerichtete Institut ist eine zentrale Einrichtung des Landes Baden-Württemberg, die für die Archive und Bibliotheken in Trägerschaft des Landes unentgeltlich Restaurierungen und Verfilmungen vornimmt. Bisher wurden 45 Werke aus unserem Bestand mit hohem Aufwand restauriert. Für jedes restaurierte Werk wird im IfE ein Restaurierungsprotokoll angefertigt. Die Restaurierungsprotokolle beschreiben den Ist-Zustand sowie die vorgenommenen Änderungen und vermerken jeden Arbeitsgang mit Angabe des Zeitaufwands sowie der eingesetzten Mittel und Verfahren. Demzufolge werden zwischen 20 und 107 Stunden für die Restaurierung jedes einzelnen Werkes aufgewandt ${ }^{31}$. Aus den finanziellen Mitteln der Bibliothek wäre dies nie zu leisten, da weder eine Personal- noch eine Kostenstelle für Restaurierungen vorhanden ist. Der hohe Aufwand macht einen gezielten Ressourceneinsatz notwendig: Wir geben nur solche Werke nach Ludwigsburg, die nach Ausweis des Verbundkatalogs in keiner anderen Bibliothek von BadenWürttemberg vorhanden sind.

Die größten Schäden sind im Bereich des Einbands und der Heftung zu verzeichnen; Papierschäden treten demgegenüber zurück. Bei Werken des 19. und 20. Jahrhunderts liegt dagegen der Schwerpunkt auf den Säureschäden. So wurden in Ludwigsburg auch fünf Werke behandelt, die erhebliche Säureschäden aufwiesen und bruchgefährdet waren ${ }^{32}$. Sie konnten durch Papierspaltung und Entsäuerung wieder stabilisiert und somit gerettet werden.

Zum Schutz wird für die restaurierten Werke in Ludwigsburg eine Kassette aus säurefreier Pappe angefertigt; seit 1999 werden sie auch verfilmt. Ein Nebeneffekt der Restaurierung ist die wieder zum Vorschein gekommene Einbandmakulatur, die auf Buchdeckel geklebt oder als schmale Streifen im Falz verborgen war. In mehreren Fällen handelt es sich sogar um karolingische Majuskelschrift!

30 Vorgänger war die Zentrale Restaurierungswerkstatt des Landes in der UB Tübingen, die für uns seit 1990 Restaurierungen vornahm.

31 Zur Restaurierung der „Konstanzer Handschrift“ vgl. Wagner, Peter Chr., Konstanzer Handschrift in neuem Glanz, in: uni-info 227 (1994) S. 20.

32 Dies sind die oben S. 27 erwähnten Bände von Aldanov sowie die Erstausgabe von Gottfried Benns „Gehirne“ (R 88/316). 
Wir geben die restaurierten Werke nicht mehr in die Fernleihe. Es wurde ein Zettel mit dem Fernleihverbot und dem Hinweis auf den Mikrofilm entworfen, der in die Bücher eingelegt wird. Der Transport der Rara nach und von Ludwigsburg erfolgte in den ersten Jahren mit dem Fernleihbücherauto - allerdings in einer speziellen Alu-Kiste. Seit dem Wegfall des Bücherautos übernimmt der Bestandserhaltungsreferent den Transport in Verbindung mit der jährlichen Bestandserhaltungsbesprechung in Ludwigsburg.

\section{Entsäuerung}

Im Rahmen des von der Landesregierung aufgelegten Landesrestaurierungsprogramms 2003/2004 wurden im Herbst des Jahres 2003 in einer größeren Aktion 369 säuregeschädigte Bücher der Rara-Sammlung sowie 480 der orientalistischen Sammlung Steige ${ }^{33}$ durch die Firma SOBU in Fürth in dem von ihr entwickelten Trockenverfahren erfolgreich entsäuert.

\section{Rara-Pflege}

Eine wichtige Maßnahme im Rahmen der Bestandserhaltung und Bestandspflege war die Reorganisation der Rara-Aufstellung, die vom Bestandserhaltungsreferenten in den Jahren 1996-1998 durchgeführt wurde. Trotz der Vorgaben für die Formattrennungen waren - bedingt durch die großen Zugangsmengen in den Aufbaujahren - zahlreiche Werke falsch eingeordnet worden, wodurch sich mit der Zeit ernste Probleme für die Stabilität ergaben. Im Extremfall standen Groß- oder sogar Liegeformate neben Klein- oder Normalformaten, so dass die Einbände sich umbogen oder herunterklappten! Oder ein Normalformat lag als unterstes unter den Liegeformaten, die dadurch in Schräglage gerieten oder sich nach unten wölbten. In manchen Regalen standen die Bücher viel zu eng, in anderen gähnten breite Lücken, wodurch die Bücher stark verzogen waren. Hieran zeigte sich, dass für die Buchpflege im Rara-Raum niemand zuständig war.

Auf Vorschlag des Bestandserhaltungsreferenten wurde ein Mitarbeiter der Benutzungsabteilung, der als gelernter Buchbinder die erforderliche Sensibilität im bestandsschonenden Umgang mit Büchern besaß, für die Aufgabe der Reorganisation sowie der ständigen Betreuung des Rara-Raums ausgewählt. Etwa 720 Werke wurden umsigniert und bei den richtigen Formatgruppen aufgestellt. Ferner wurde die Regalbefüllung korrigiert und im Austausch zu

33 Zur Sammlung Steiger vgl. Steffen, Reinhild - Wagner, Peter Christoph, Alf laila walaila. Tausendundeine Nacht. Die Schleier sind gelüftet, in: Bibliothek aktuell 64 (1994) S. 12-21 = http://www.uni-konstanz.de/ZE/Bib/ba/ba646.htm; Wagner, Peter Chr., Nicht nur „Tausendundeine Nacht“. Die orientalistische Sammlung Steiger, in: uni-info 228 (1994) S. 1-4. 
vorhandenen Bücherstützen Klemmbügel, die am oberen Regalboden einzuhängen sind, zur Stützung verteilt; schmale Broschüren mit fragilem Papieroder Pappeinband wurden in Ziehmappen aus säurefreier Pappe geborgen; desgleichen Werke mit brüchigem Einband. Bücher mit Schließen oder Einbandrelief wurden zwischen zwei Pappen gestellt, um beim Herausnehmen oder Zurückstellen die Beschädigung der benachbarten Bücher zu vermeiden; die Liegeformate wurden mit abnehmender Größe von unten nach oben angeordnet.

Im Rahmen der innerbetrieblichen Weiterbildung hielt der Bestandserhaltungsreferent Ende 1996 einen Vortrag über den Umgang mit den Rara und erläuterte anhand von beschädigten Werken die Schadensursachen und die Möglichkeiten der Vermeidung. Dieser Vortrag ist auch als Video vorhanden ${ }^{34}$. Die seit 1996 jährlich stattfindende Bestandserhaltungsbesprechung im Institut für Erhaltung von Archiv- und Bibliotheksgut in Ludwigburg dient der Weiterbildung des Bestandserhaltungsreferenten. Außerdem führte das Institut im Jahre 2001 eine landesweite Fortbildungstagung für Magazinmitarbeiter durch, an der auch zwei Mitarbeiterinnen aus Konstanz teilnahmen.

\section{Aktivitäten}

\section{Ausstellungen}

Einen besonderen Platz unter den Aktivitäten nehmen die Ausstellungen ein:

Eine Ausstellung „Kräuterbücher aus zwölf Jahrhunderten“ veranstaltete Dr. Laurenz Bösing im Jahre 1986. Dabei präsentierte er Faksimiles aus dem Rara-Bestand sowie Originale aus der Suso-Bibliothek.

Die reichen Bestände an Faksimile-Zeichnungen Leonardo da Vincis ermöglichten Michael Sukale im Jahre 1987 eine große Ausstellung ${ }^{35}$, die sogar andernorts gezeigt wurde, nämlich in Friedrichshafen und in Trier ${ }^{36}$.

Eine Ausstellung zu Annette von Droste-Hülshoff und ihrem literarischen Umfeld wurde 1988 vom damaligen Fachreferenten für Germanistik, Dr. Robert Bergmann, veranstaltet, der bei der Eröffnung auch einen einleitenden Vortrag hielt ${ }^{37}$.

34 Wagner, Peter Chr., Umgang mit Rara (Konstanz 1997).

35 Sukale, Michael, Sehen als Erkennen - Leonardo da Vincis Zeichnungen in Faksimile (=Ausstellungskataloge Universitätsbibliothek Konstanz; 8a) (1987).

36 Sukale, Michael: Sehen als Erkennen - wissenschaftliche Zeichnungen von Leonardo da Vinci (=Ausstellungskataloge Universitätsbibliothek Konstanz; 8b) (1987).

37 Bergmann, Robert, Annette von Droste-Hülshoff und ihr literarisches Umfeld (=Ausstellungskataloge Universitätsbibliothek Konstanz; 16) (1988). 
Mit den zahlreichen Faksimiles mittelalterlicher Bibeln konnte eine Ausstellung bestückt werden, die mit dem Titel „Biblia“ im Jahre 1994 von den Praktikantinnen und Praktikanten veranstaltet wurde.

Ebenfalls mit den Faksimiles konnte Maria Herkner von der Arbeitsgruppe Kunstwissenschaft im Rahmen eines Forschungsprojektes der Universität 1995 eine Ausstellung zur Reichenauer Buchmalerei veranstalten ${ }^{38}$.

Seit 1994 gibt es eine ständige Rara-Vitrine im zentralen Eingangsbereich der Bibliothek mit jährlich ein bis zwei wechselnden Ausstellungen, wobei jeweils zwischen ein und drei Büchern ausgestellt werden. Diese Ausstellungen werden vom Bestandserhaltungsreferenten durchgeführt ${ }^{39}$. Auf einer Texttafel sind die wichtigsten Angaben zu Autor und Werk sowie zur Typographie und zum Buchschmuck zusammengestellt. Dieser Text wird jeweils auf der Homepage der Bibliothek unter „Ausstellungen“ ins Internet gestellt ${ }^{40}$. Zeitnah erscheint er auch - z.T. mit Bildern - als Artikel in unserer Hauszeitschrift „Bibliothek aktuell“41 sowie im Uni-Magazin „uni’kon““42, das bis 2001 „uniinfo“43 hieß. In einigen Fällen hat die Pressestelle der Universität zu diesen Ausstellungen auch eine Presseerklärung herausgegeben. In deren Folge kam es im Mai 1994 sogar zu einem Interview mit dem Bodensee-Radio über unsere „Konstanzer Handschrift“.

Diese im Sinne einer Öffentlichkeitsarbeit betriebenen Aktivitäten sollen die Schätze unseres Altbestands einer breiteren Öffentlichkeit bekannt machen. Daher wurde ganz bewusst der Standort für die Vitrine gegenüber den Verbuchungstheken gewählt. Aus konservatorischer Sicht ergibt sich ein Konflikt mit der Lichtexposition, die hier im Eingangsbereich natürlich heller ist, als es bei Ausstellungen alter Bücher geboten ist. Um dem Rechnung zu tragen, erfolgt ein monatlicher Seitenwechsel.

\section{Vorträge und Führungen}

Die „Konstanzer Handschrift“ wurde nach ihrer Restaurierung und Ausstellung der "Gesellschaft der Freunde und Förderer der Universität Konstanz e.V." in einer Sitzung präsentiert.

38 Herkner, Maria, Reichenauer Buchmalerei. Faksimiles und ihre Vorläufer (=Ausstellungskataloge Universitätsbibliothek Konstanz; 22) (1995).

39 Vgl. Wagner, Peter Chr., Rara-Austellung, in: Bibliothek aktuell 66 (1995) S. 19-21.

40 http://www.ub.uni-konstanz.de/Ausstellungen.htm

41 zuletzt in Heft 77, 2002: http://www.ub.uni-konstanz.de/kops/volltexte/2002/900/

42 http://www.uni-konstanz.de/struktur/campus/unikon/

43 http://www.uni-konstanz.de/struktur/campus/info/uniinfo.html 
Anlässlich der Ausstellung der gesponserten Hus-Flugschrift „Geistlicher Bluthandel Johannis Hussz"“44 hielt Prof. Alexander Patschovsky vor der RaraVitrine einen Vortrag.

Im Rahmen der regionalen Öffentlichkeitsarbeit entstand in der Fachreferentensitzung die Idee, mit einer Vortragsreihe aus der Universität herauszugehen und einem städtischen Publikum unsere Schätze nahe zu bringen. So sollte der Bekanntheitsgrad der Konstanzer Bestände gesteigert werden. Im Wintersemester 2000/01 wurden unter dem Titel "Schätze aus der Bibliothek“ die fünf folgenden Vorträge im Kulturzentrum der Stadt Konstanz anhand von Originalen oder von Dias veranstaltet: „Reichenauer Buchmalerei“ (lic.phil. Christina Egli, Bibliothekarin), „Konstanzer Handschrift aus der Zeit des Konzils“ (Dr. Laurenz Bösing, ehemaliger Konstanzer Fachreferent, der diese Handschrift 1985 erworben hatte), „Frühe Reiseführer aus der Bodenseeregion“ (Werner Allweiss M.A., Fachreferent für Geschichte), „Schätze aus dem Rara-Bestand" (Dr. Peter Christoph Wagner, Bestandserhaltungsreferent und Fachreferent), „Die „Gutenberg-Bibel und die Erfindung des Buchdrucks“ (Dr. Uwe Jochum, Fachreferent für Germanistik). Mit in der ganzen Stadt sowie in der Universität ausgehängten Plakaten wurde für diese Vortragsreihe geworben. Die Zuhörerzahlen schwankten zwischen 20 und 40. Angesichts der Konkurrenz zu zahlreichen parallelen Kulturveranstaltungen konnte sich unsere Aktion damit ganz gut behaupten. Im Zusammenhang mit der Vortragsreihe fand ein Interview mit einer Reporterin des "Südkurier" statt, der bei einem Rundgang durch den Rara-Raum Konzeption und Bestandsumfang vermittelt sowie einige ausgewählte Schätze präsentiert wurden. Der entsprechende Zeitungsartikel erschien einen Tag vor dem Rara-Vortrag. Dies trug sicherlich dazu bei, dass dieser Vortrag der bestbesuchte war.

Eine Fortsetzung fand diese Vortragsreihe im Sommersemester 2002 mit folgenden Vorträgen, die nunmehr im Seniorenzentrum gehalten wurden:

„Biblia pauperum - die Armenbibel“ (Christina Egli), „Die Exultet-Rolle“ (Dr. Adalbert Kirchgäßner), „Die Gutenberg-Bibel“ (Dr. Uwe Jochum), „Die Encyclopédie von Diderot und d'Alembert" (Friedrich E. Dahlmann, Dipl.Bibliothekar).

Der Vortrag „Schätze aus dem Rara-Bestand“ wurde zu einem kursorischen Überblick über die Konstanzer Rara-Sammlung erweitert und unter diesem Titel ins Internet gestellt ${ }^{45}$. Diese Präsentation wird laufend erweitert, während

44 vgl. oben S. 4.

45 http://www.ub.uni-konstanz.de/fi/rara/index.htm

BIBLIOTHEKSDIENST 38. Jg. (2004), H. 1 
eine zweite Fassung als Pdf-Dokument auf KOPS liegt, dem Server der elektronischen Publikationen ${ }^{46}$.

Die öffentliche Feier mit der Übergabe von Mareys „La méthode graphique“ und dem Vortrag von Dr. Steffen Bogen wurde oben bereits erwähnt ${ }^{47}$.

Als im Dezember 2002 der i.@day stattfand, der Informationstag der Bibliothek, der mittels Aktionen, Veranstaltungen, Vorträgen und Führungen der universitären Öffentlichkeit die Bibliothek als den kompetenten Partner für Informationsvermittlung vorstellen sollte, gab es auch drei Rara-Vorträge zu den „wertvollsten Büchern der Bibliothek“, die zu den bestbesuchten Veranstaltungen dieses Tages gehörten ${ }^{48}$ !

Führungen im Rara-Raum sind eingeschränkt möglich. Wir bieten sie eigentlich nur auswärtigen Gästen der Bibliothek an. Auch im Rahmen der Ausbildung der Referendare zum höheren Bibliotheksdienst findet eine Führung statt. Ausnahmsweise wurde die Josua-Rolle (R 55/175) einige Male dem Leistungskurs Latein des Gymnasiums in Tettnang präsentiert. Im Rahmen von Volkshochschulführungen stellt Friedrich E. Dahlmann seit 1983 ausgewählte Rara vor, die er auf einem Tisch vor dem Rara-Raum deponiert.

Trotz der geschilderten Aktivitäten darf nicht übersehen werden, dass eine kontinuierliche systematische Arbeit am alten Buch nicht institutionalisiert ist. Lediglich die Exlibris wurden erfasst ${ }^{49}$. Dagegen fehlt z.B. eine Erfassung von Besitzvermerken oder Widmungen, die für eine Überlieferungs- und Herkunftsgeschichte der Bände unerlässlich wäre. Eine Suetonausgabe, 1646 in Amsterdam erschienen (R 58/531), sei nur als Beispiel für gleich fünf Besitzvermerke angeführt.

\section{Dienstleistungen}

Die Aktivitäten der Bibliothek Konstanz betreffen nicht nur den eigenen Bestand, sondern auch Dienstleistungen für andere Bibliotheken mit Altbeständen: Für die Bearbeitung der seit 1604 bestehenden Bibliothek des HeinrichSuso-Gymnasiums, bei der es sich großenteils um die Bestände des ehemaligen Jesuitenkollegs handelt, boten wir einen EDV-gestützten Arbeitsplatz, an dem - mit wechselnden Personen - jeweils eine Diplom-Kraft eingearbeitet

46 http://www.ub.uni-konstanz.de/kops/volltexte/2001/645/

47 vgl. oben S. 4

48 Wagner, Peter Chr., Rara-Vortrag am i.d@y, in: Bibliothek aktuell 78, 2003, S. 7 = http://www.ub.uni-konstanz.de/kops/volltexte/2003/1025/

49 Exlibris in Büchern der Bibliothek der Universität Konstanz; hrsg. von Gerhard Schmitz-Veltin, mit Beiträgen von Dietrich Wehner und Brian Nee Lee (1991) 
wurde und von 1988-1997 den etwa 24.000 Bände umfassenden Bestand im Verbundkatalog katalogisieren konnte ${ }^{50}$. Außerdem wurden von den Fachreferenten sachlich-systematische Notationen vergeben. Damit sind diese wertvollen historischen Bestände formal und inhaltlich nachgewiesen sowie überregional recherchierbar. Es ist daher fast eine logische Konsequenz, dass die Benutzung der Suso-Bibliothek seitdem über die UB Konstanz organisiert ist.

Darüber hinaus bieten wir seit 2001 für die von der Bibliothek gemeinsam mit Prof. Alexander Patschovsky initiierte und von der DFG geförderte Katalogisierung der mittelalterlichen Handschriften des Suso-Gymnasiums durch die Konstanzer Mediävistin Dr. Brigitte Hotz den EDV-Arbeitsplatz sowie mit unserem buchhistorischen Bestand die Arbeitsmöglichkeiten für die ErschlieBung. Es handelt sich um 40 Handschriften sowie 50 Handschriftenfragmente mit Schwerpunkt im theologischen, liturgischen und juristischen Bereich.

Für die Katalogisierung der Altbestände der Leopold-Sophien-Bibliothek in Überlingen, die etwa 30.000 Bände umfassen ${ }^{51}$, leisteten wir Mitte der 90er Jahre Hilfestellung: eine Diplom-Bibliothekarin unserer Bibliothek nahm 1996 die Einarbeitung der Überlinger Bibliothekarin in die elektronische Katalogisierung vor und katalogisierte auch selbst eine größere Zahl von Büchern.

Die städtische Wessenberg-Bibliothek, die Privatbibliothek des Konstanzer Bistumsverwesers Freiherr von Wessenberg ${ }^{52}$, die zuvor in beengten Verhältnissen im Keller eines Konstanzer Gymnasiums aufgestellt war, wurde nach Verhandlungen zwischen Universität und Stadt als Leihgabe in die Obhut der Universitätsbibliothek überführt und im Juni 2001 in den Räumen der UB in einem vom Freihandbereich abgeschlossenen klimatisierten Magazin aufgestellt. Wir bieten auch in diesem Fall einen EDV-Arbeitsplatz für die Katalogisierung der Bestände, die zur Zeit noch läuft. Im Rahmen der Rara-Benutzung ermöglichen wir auch die Benutzung der Wessenberg-Bestände.

Zur besseren Erschließung der Bestände wurde 1994 ein elektronischer Regionalkatalog konfiguriert, der mit den Daten aus dem Verbundkatalog des

50 Zur Suso-Bibliothek vgl. Hesse, Hans, in: Handbuch der historischen Buchbestände in Deutschland Bd. 8 (1994) S. 101-106; von Bohr, Helmut, Die Lehrerbibliothek im Heinrich-Suso-Gymnasium Konstanz und das Projekt der maschinenlesbaren Erfassung ihrer Bestände, in: Bibliothek aktuell 60 (1991) S. 4-6; ders., Die Jesuitenbibliothek im Heinrich-Suso-Gymnasium in Konstanz, in: Bibliothek aktuell 70 (1997), S. 42-44 = http://www.uni-konstanz.de/ZE/Bib/ba/ba7011.htm

51 bezogen auf die Bücher vor 1900. - Zur Leopold-Sophien-Bibliothek vgl. http://www.ub.uni-konstanz.de/regio/lsbueb.htm

52 Ausführlich zu Geschichte, Bestand, weiterführender Literatur: Handbuch der historischen Buchbestände in Deutschland Bd. 8 (1994) S. 107-114; „WessenbergBibliothek": http://www.ub.uni-konstanz.de/wessenberg.htm 
Südwestdeutschen Bibliotheksverbundes gespeist wird ${ }^{53}$. Er enthält die Bestände von dreizehn Bibliotheken des Bodenseeraumes, darunter auch die Suso-Bibliothek, die Wessenberg-Bibliothek und die Leopold-SophienBibliothek.

\section{Benutzung}

inneruniversitär

Die Werke aus dem Rara-Bestand werden auf Wunsch in den Lesesaal der Mediothek unter Aufsicht zur Benutzung ausgegeben. Zur Bestandsschonung wird empfohlen, die Werke auf Schaumstoff-Keilkissen, mit Unterfütterung durch einzelne Keile, zu legen, um den Druck der aufgeschlagenen Deckel auf den Rücken und die Bindung zu entlasten. Die bereitgelegten Baumwollhandschuhe, die uns von einer Dozentin gespendet wurden, werden allerdings kaum genutzt. Dem zunehmend häufiger geäußerten Wunsch nach Reproduktionen konnte seit Beginn des Jahres 2003 mit der Inbetriebnahme des buchschonenden Scanners „Bookeye“ Genüge geleistet werden. Bei diesem erfolgt der Scanvorgang mit UV- und IR-freien Kaltlichtlampen von oben; und das Buch muß nicht einmal ganz aufgeschlagen werden. Die Lichtstärke von 3000 Lux ist unter dem Gesichtspunkt der Bestandserhaltung vertretbar. Allerdings wird wegen der Wärmeentwicklung darauf geachtet, dass nicht mehr als 20 Scans auf einmal gemacht werden.

Die Nachfrage nach Rara ist mit etwa 10 Benutzern pro Woche recht zufrieden stellend.

Gelegentlich wird von Dozenten die Präsentation von Rara in ihren Lehrveranstaltungen gewünscht. Aus konservatorischen Gründen entsprechen wir dem nicht, sondern verweisen darauf, dass das betr. Werk einzeln im Lesesaal eingesehen werden kann. Hier macht sich das Fehlen eines eigenen Vortragsraumes negativ bemerkbar.

Im allgemeinen entzieht sich die inhaltliche Verwendung eines benutzten Rara-Werkes unserer Kenntnis. Dennoch können einige „Highlights“ hervorgehoben werden:

Anja Grebe konnte sich für ihre Dissertation „Die Ränder der Kunst. Buchgestaltung in den burgundischen Niederlanden nach 1470“ (Konstanz 2000) auch auf die Faksimile-Sammlung mittelalterlicher Buchmalerei in unserem Rara-Bestand stützen.

53 http://www.ub.uni-konstanz.de/regio/ 
Der „Ulysses“" ${ }^{\text {54 }}$ wurde bereits bald nach seiner Erwerbung von Prof. Aleida Assmann, Anglistik, in ihre Vorlesung im Wintersemester 2000/01 einbezogen.

Prof. Hartmut Riehle, Sportwissenschaften, hat sich im Rahmen der Ausarbeitung eines Kongressvortrages über die Geschichte der Biomechanik eingehend mit dem Werk „De motu animalium“ von Giovanni Alfonso Borelli beschäftigt, das wir in der Ausgabe Neapel 1734 besitzen (R 73/138).

Marey's "La Méthode graphique" nahm in Steffen Bogens und Joachim Paechs interdisziplinären Seminar „Repräsentierte Zeit und Bewegung in Bildern und Diagrammen: Mittelalter/Moderne“ im Sommersemester 2003 einen zentralen Platz ein.

Das Filmexperiment „Anémic cinéma“ von Marcel Duchamp ${ }^{55}$ verwendete Friederike Wappler in ihrem Foucault-Seminar im Sommersemester 2003.

\section{überregionale Nutzung}

Die überregionale Nutzung erfolgt schwerpunktmäßig im Rahmen der konventionellen Fernleihe: Wir geben unsere Rara auf Anforderung an auswärtige Bibliotheken. In der Gewährung sind wir eigentlich sehr liberal. Es kommen sogar Direktbestellungen im Rahmen von „Subito“; diese lehnen wir allerdings ab.

Punktuell sind folgende Aktionen im Rahmen überregionaler Nutzung zu nennen:

Die „Konstanzer Handschrift“ wurde anlässlich des Jubiläums „40 Jahre Baden-Württemberg“ im Jahre 1992 auf einer Sonderausstellung gezeigt.

Unsere Erstausgabe von Johann Heinrich Voß' llias-Übersetzung aus dem Jahre 1793 (R 96/85) wurde für die viel beachtete und umstrittene TrojaAusstellung „Troia - Traum und Wirklichkeit“ im Jahre 2201 als Leihgabe angefordert und auch vergeben.

Der Verlag K.G.Saur nahm eines unserer Werke, und zwar die „Reisen in Arabien" von Johann Ludwig Burckhardt (1830 - Sign.: R 81/40), in sein Projekt der Volltext-Mikrofiche-Edition „Deutschsprachige Schriften zum Islam vom 16. Jahrhundert bis $1900^{56}$.

54 vgl. oben S. 8.

55 vgl.oben S.2.

56 Deutschsprachige Schriften zum Islam vom 16. Jahrhundert bis 1900. Teil 1: Religion und Theologie, Recht und Sitte (2003) Fiche Nr. 052-056. 
Der Internettext zur Ausstellung von Al-Farganis „Chronologica et astronomica elementa" ${ }^{457}$ fand wegen der Erwähnung einer Sonnenfinsternis das Interesse von Franz Krojer aus München. Dieser schrieb ein Buch zur mittelalterlichen Chronologie, gestützt auf die astronomischen Daten und ihre Erwähnung in den historischen Quellen ${ }^{58}$. In diesem Buch hat Krojer den gesamten Text zu Al-Fargani übernommen ${ }^{59}$. Krojer geht es mit seinem Buch insbesondere um eine Widerlegung der Thesen Heribert Illigs vom angeblichen Fehlen des frühen Mittelalters.

Auswärtige Anfragen kommen eher selten vor: Bezüglich des Werkes „De I'esprit" von Claude Adrien Helvétius (Paris 1758 - R 73/77) wollte David W. Smith, Toronto, wissen, ob wir ein Exemplar der ersten oder der zweiten Druckvariante der Erstausgabe oder der zweiten Auflage haben. Anhand des Anfangswortes ausgewählter Zeilen ließ sich eine Zuordnung zur zweiten Variante der Erstauflage vornehmen. Smith stellte in langjährigen Forschungen zur komplizierten Editionsgeschichte die erste Bibliographie der Werke des Philosophen Helvétius zusammen ${ }^{60}$.

Die Johann-Gerhard-Forschungsstelle am Theologischen Seminar der Universität Heidelberg interessierte sich im Rahmen der Rekonstruktion der Bibliothek des Jenaer Theologieprofessors Johann Gerhard (1582-1637) für unser Buch „Antiquarum lectionum commentarii“ von Lodovico Ricchieri (Paris 1517 - R 56/1205), weil dieses Werk im Katalog der Gerhardschen Bibliothek verzeichnet ist. Wir übermittelten ein Foto des Titelblatts sowie eines der letzten Seite. Inzwischen ist die Publikation dieses Forschungsvorhabens erschienen $^{61}$.

57 http://www.ub.uni-konstanz.de/fi/rara/index.htm\#1200_Jahre_Al-Fargani

58 Krojer, Franz: Die Präzision der Präzession. Illigs mittelalterliche Phantomzeit aus astronomischer Sicht (München 2003).

59 Krojer a.O. S. 203-206.

60 Smith, David W., Bibliography of the writings of Helvétius (Paris 2001).

61 Bibliotheca Gerhardina. Rekonstruktion der Gelehrten- und Leihbibliothek Johann Gerhards (1582-1637) und seines Sohnes Johann Ernst Gerhard (1621-1668), hrsg. von J.A. Steiger, 2 Bde. Stuttgart-Bad Cannstatt 2002. - Ricchieris Kommentar ist auf S. 1015, Nr. R108/447-1 erfasst. 